\title{
ESTUDO DO MOMENTO ÓTIMO DE REFORMA PARA POVOAMENTOS DE Eucalyptus spp - O CASO DA REDUÇÃO DOS CUSTOS
}

\section{STUDY OF THE OPTIMAL TIME FOR REPLACEMENT OF Eucalyptus spp. PLANTATIONS - THE CASE OF COSTS REDUCTION}

\author{
Álvaro Nogueira de Souza ${ }^{1}$ Antônio Donizette de Oliveira ${ }^{2}$ José Luiz Pereira de Rezende ${ }^{2}$
}

\begin{abstract}
RESUMO
Os objetivos deste trabalho foram: a) propor e verificar a efici ência de um modelo matemático que permita estudar os efeitos do progresso tecnológico (redução dos custos) sobre a época ótima de substituir povoamentos de Eucalyptus spp; b) determinar a rotação florestal de um povoamento de Eucalyptus spp; c) verificar qual seria o momento de substituir se as receitas estivessem constantes desde o in ício dos incentivos fiscais, ou seja, na década de $60, \mathrm{e}, \mathrm{d}$ ) projetar o momento de substituir o povoamento no futuro, considerando os custos decrescentes e a produtividade constante a valores de hoje. Utilizou -se a Função Gompertz para o cálculo da produção de madeira. O critério utilizado para a avaliação econômica da viabilidade dos projetos foi o Valor Presente Líquido. O modelo proposto permitiu calcular o efeito do progresso tecnológico nos custos no período entre 1960 e 2000 e projetar os valores dos custos para o período entre 2000 e 2040. Concluiu-se que: a) a rotação, com valores atuais, encontra-se, em 7 anos, mantidas as receitas da década de 60; b) o momento ótimo de reforma estaria em seis cortes; c) o modelo mostrou-se eficiente para estimativas em um horizonte de até 40 anos; o estudo da cadeia de substituição mostrou que as épocas ótimas de reforma caíram ao longo dos anos, de seis cortes na década de 60, três na década de 80 e dois atualmente; a tendência é permanecer nos dois cortes passando a três com um pequeno aumento na produtividade das talhadias.
\end{abstract}

Palavras-chave: progresso tecnológico, reforma de Eucalyptus, custos florestais.

\begin{abstract}
The objectives of this work were: a) to study the effects of cost reduction on the optimal replacement time of Eucalyptus plantations; b) to determine rotation age, keeping income constant and cost decreasing overtime; c) to verify the efficiency of a mathematical model for modelling technological progress effects; d) to verify which would be the substitution cicle if the income had been constant since the sixties; and, e) to project substitution time in the future, considering decreasing cost and constant income at today's values. Data from forest enterprises located in the Cerrado region were used. The Gompertz Function was employed to calculate timber yield. The economical evaluation method used was the Present Net Worth. The proposed model allowed calculating technological progress effect on costs in the 1960 2000 period and to project the cost values for the 2000 - 2040 period. It was concluded that: a) the rotation, with current values is 7 years; b) maintaining the income at the sixties levels, the optimal substitution cycle would be at 6 cuttings; c) the model proved to be efficient for estimates of up 40 years horizon; and, d) the substitution chain showed that the optimal substitution cycle went down along the years, from 6 cuttings in the sixties, 3 in the eighties and 2 currently; the future tendency is to stick with 2 cuttings, reaching 3 cuttings with increase in the coppice yields.
\end{abstract}

Key words: technological progress, renewal, forest costs.

\section{INTRODUÇÃO}

O caminho natural de qualquer empreendimento, que busque a maximização do lucro sobre o capital investido, passa obrigatoriamente por pesquisas em que o aumento da produtividade e a redu ção dos custos são os objetivos principais. A tendência atual, no Setor Florestal, é trabalhar na redução dos custos, uma

1. Engenheiro Florestal, Professor da Faculdade de Engenharia Florestal, Fundação Integrada Municipal de Ensino Superior (FIMES), Mineiros (GO). Doutorando em Engenharia Florestal pela Universidade Federal de Lavras, Rua Aníbal Teodoro, 110, Bairro Pitanguí, CEP 37200-000, Lavras (MG). ansouza@ufla.br

2. Engenheiro Florestal, Professor do Departamento de Ciências Florestais, Universidade Federal de Lavras, Campus Universitário, CEP 37200-000, Lavras (MG). 
vez que a produtividade dos plantios atuais já alcança altos índices e, qualquer ganho nesse campo, será pequeno, principalmente para o caso do estado de Minas Gerais onde os plantios se localizam em áreas com capacidade produtiva limitada.

Nesse sentido, procurar conhecer o comportamento dos custos em um empreendimento florestal é uma das variantes mais importantes no caminho que leva a decis ões em relação à questão da reforma ou substituição de um povoamento que, ao longo dos anos, vem sofrendo modificações nos seus custos, dado um progresso tecnológico evidente dentro do Setor.

Steindl (1980) considerou progresso tecnológico como um processo que acarreta, a longo prazo, o aumento do produto por trabalhador, e que possa experimentalmente ser medido por ele. $\mathrm{O}$ autor mostrou que o progresso tecnológico pode proporcionar a economia do fator terra e recursos naturais, e conseqüentemente, de capital, além de produzir novos bens e afetar a qualidade de vida. Uma outra característica é o estímulo ao investimento.

Dentre as atividades de manejo, a reforma é uma das mais importantes, porque proporciona a continuidade da atividade florestal. Silva (1990) definiu reforma como sendo a substituição total do povoamento de baixo potencial produtivo, ou com produtividade abaixo da esperada, por um novo povoamento originado do plantio de mudas.

Rezende et al. (1987) comparou um povoamento florestal a uma máquina que produz o produto madeira, chamando a atenção para o fato de que a época ótima de substituir a floresta poderia ser encontrada da mesma maneira que se encontrava a época de substituir uma máquina qualquer.

De acordo com os conceitos da Engenharia Econômica, Massé (1962) diferenciou substituição de reforma quando se trata de máquinas e equipamentos. Para ele, uma substituição é efetuada quando o equipamento se torna incapaz de exercer a fun ção para a qual foi designado e construído, e reforma seria um "reparo" no equipamento para que continue a produzir dentro do esperado.

Rezende et al. (1987) definiram reforma e substituição para o caso de um povoamento florestal, como sendo a substituição considerada, quando o povoamento não produz de maneira satisfatória. Já a reforma se refere a uma situação na qual um povoamento ainda é capaz de executar suas funções, mas pode ser alterado ( interplantio, adensamento) ou mesmo ser substitu ído por outro mais eficiente.

Os objetivos deste trabalho foram: a) propor e verificar a eficiência de um modelo matemático que permita estudar os efeitos do progresso tecnológico (redução dos custos) sobre a época ótima de substituir povoamentos de Eucalyptus spp; b) determinar a rotação florestal de um povoamento de Eucalyptus spp; c) verificar qual seria o momento de reforma se as receitas estivessem constantes desde o in ício dos incentivos fiscais, ou seja, na década de 60; projetar o momento de substituir o povoamento no futuro, considerando os custos decrescentes e a produtividade constante a valores de hoje.

\section{MATERIAL E MÉTODOS}

O método de análise econômica utilizado foi o Valor Presente Líquido, considerando a replicação do projeto para um horizonte infinito $\left(\mathrm{VPL}_{\infty}\right)$ que, segundo Rezende e Oliveira (2001), é dado por:

$$
V P L_{\infty}=\frac{V P L(1+r)^{n t}}{(1+r)^{n t}-1}
$$

Em que:

$$
V P L=\sum_{x=0}^{n t} R_{x}(1+r)^{-x}-\sum_{x=0}^{n t} C_{x}(1+r)^{-x}
$$

Em que: $\mathrm{C}_{\mathrm{x}}=$ custos efetuados no ano $\mathrm{x} ; \mathrm{R}_{\mathrm{x}}=$ receitas auferidas no ano $\mathrm{x} ; \mathrm{r}=$ taxa anual unitária de desconto; $\mathrm{t}=$ rotação em anos; $\mathrm{n}=$ número de cortes; $\mathrm{x}=$ ponto no tempo, em anos, em que ocorrem custos e receitas.

Para estimar a produção de madeira, foi utilizada a Função Gompertz que é dada por: 


$$
Y=K\left(1-e^{-a \cdot e^{b \cdot m}}\right)
$$

Em que: $\mathrm{K}$, a e $\mathrm{b}=$ coeficientes; $\mathrm{m}=$ Idade do povoamento em meses; $\mathrm{e}=$ Base dos logaritmos neperianos; $\mathrm{Y}=$ Produção de madeira em st/ha. forma:

Os custos que ocorrem durante um ciclo de produção de madeira podem ser divididos da seguinte

- Custos iniciais: são aqueles que ocorrem no ano zero do projeto, ou seja, no ano de sua implantação. Podem ser subdivididos em preparo da área, preparo do solo, controle de pragas, capina química, adubação, produção de mudas, plantio, supervisão técnica e diversos.

- Custo de manutenção florestal: são os necessários para manter a floresta, com ocorrência ano 1 ao ano de corte. Porém, nem todos se repetem ao longo dos anos. Tais custos estão subdivididos em tratos culturais, adubação, controle de pragas, controle de incêndios florestais, inventário florestal, supervisão técnica, custo da terra, bateção pré-corte, colheita e diversos.

- Custos da regeneração florestal: são os gastos que as empresas têm para conduzir a brotação dos plantios originais para uma ou mais talhadias. Podem ser subdivididos em tratos culturais, adubação, controle de pragas, controle de incêndios florestais, inventário florestal, supervisão técnica, custo da terra, bateção pré-corte, colheita e supervisão técnica.

A planilha de custos utilizada para os cálculos está representada na Tabela 1. Os custos foram divididos em três grupos. O primeiro grupo foi composto pelos custos operacionais de plantio (ano zero), o segundo grupo foi composto por custos operacionais da manutenção florestal ( $1^{\circ}$ ano ao ano de corte) e o terceiro, pelos custos operacionais da regeneração florestal ( $1^{\underline{0}}$ ano após o corte ao ano de corte das talhadias). Além desses custos, foram considerados os seguintes valores:

- Taxa de desconto: $8 \%$ ao ano;

- preço atual da madeira: US\$15.00/st;

- preço da madeira na década de 60: US\$12.00/st;

- produtividade das talhadias ( $\beta$ ): $90 \%$ do volume anterior;

- custo de colheita na década de 60: US\$6.00/st;

- custo atual de colheita: US\$4.00/st.

- custo de bateção pré-corte na década de 60: US\$48.00/ha;

- custo atual de bateção pré-corte: US\$16.00/ha.

Os custos, ao longo do tempo, precisam ser estudados de maneira diferente do que as receitas, pois enquanto estas ocorrem somente a cada rotação, aqueles ocorrem ao longo de todo o horizonte de planejamento.

\section{RESULTADOS E DISCUSSÃO}

\section{Rotação ou idade ótima de corte}

Toda base de cálculo foi elaborada com base no prévio conhecimento da idade ótima de corte, pois quando se altera qualquer variável, tem-se, quase sempre, alterações nas condições técnicas e econômicas da rotação. Portanto, é necessário recalcular a idade de corte e verificar seu efeito no número de cortes que antecedem a substituição. A Tabela 2 mostra o $\mathrm{VPL}_{\infty}$ e o volume para diversas idades para uma taxa de desconto de $8 \%$ a.a., considerando os custos e as produtividades atuais. Os volumes foram calculados pela equação (1), usando-se os coeficientes $K=300$ st/ha, $a=-0,07849, b=0,037$, conforme Souza (1999). 
TABELA 1: Custos de plantio, manutenção e regeneração de povoamentos de eucalipto de grandes empresas que atuam na área de Cerrado no estado de Minas Gerais.

\begin{tabular}{|c|c|c|c|}
\hline Custos operacionais de plantio & $\begin{array}{c}\text { Custo efetivo década } \\
\text { de } 60 \text { (US\$/ha) }\end{array}$ & $\begin{array}{l}\text { Custo efetivo } \\
\text { atual (US\$/ha) }\end{array}$ & $\begin{array}{c}\text { Ano de } \\
\text { ocorrência }\end{array}$ \\
\hline Preparo da área & 331.11 & 110.37 & 0 \\
\hline Capina química & 134.19 & 44.73 & 0 \\
\hline Adubação & 192.60 & 64.20 & 0 \\
\hline Produção de mudas & 357.54 & 119.18 & 0 \\
\hline Plantio & 87.72 & 29.24 & 0 \\
\hline Diversos & 696.84 & 232.28 & 0 \\
\hline Custos operacionais da manutenção florestal & $\begin{array}{c}\text { Custo efetivo década } \\
\text { de } 60(\mathrm{US} \$ / \mathrm{ha})\end{array}$ & $\begin{array}{l}\text { Custo efetivo } \\
\text { atual (US\$/ha) }\end{array}$ & $\begin{array}{c}\text { Ano de } \\
\text { ocorrência }\end{array}$ \\
\hline \multirow[t]{3}{*}{ Tratos culturais } & 253.95 & 84.65 & 1 \\
\hline & 95.61 & 31.87 & 2 \\
\hline & 16.08 & 5.36 & 3 a t \\
\hline \multirow[t]{2}{*}{ Adubação } & 98.97 & 32.99 & 1 \\
\hline & 16.62 & 5.54 & 2 \\
\hline Controle de pragas & 24.63 & 8.21 & $1 \mathrm{at}$ \\
\hline Controle de incêndios florestais & 10.20 & 3.40 & $1 \mathrm{at}$ \\
\hline Inventário florestal & 6.15 & 2.05 & $1 \mathrm{at}$ \\
\hline \multirow[t]{3}{*}{ Diversos } & 61.05 & 20.35 & 1 \\
\hline & 22.17 & 7.39 & 2 \\
\hline & 8.85 & 2.95 & 3 a t \\
\hline Custos operacionais da regeneração florestal & $\begin{array}{l}\text { Custo efetivo década } \\
\text { de } 60 \text { (US\$/ha) }\end{array}$ & $\begin{array}{l}\text { Custo efetivo } \\
\text { atual (US\$/ha) }\end{array}$ & $\begin{array}{c}\text { Ano de } \\
\text { ocorrência } \\
\text { (após o corte) }\end{array}$ \\
\hline \multirow[t]{2}{*}{ Tratos culturais } & 60.63 & 20.21 & 1 \\
\hline & 123.33 & 41.11 & 2 \\
\hline Adubação & 561.06 & 187.02 & 1 \\
\hline \multirow[t]{2}{*}{ Controle de pragas } & 37.56 & 12.52 & 1 \\
\hline & 24.63 & 8.21 & $2 \mathrm{at}$ \\
\hline Controle de incêndios florestais & 10.20 & 3.40 & $1 \mathrm{at}$ \\
\hline Inventário florestal & 6.09 & 2.03 & $1 \mathrm{at}$ \\
\hline \multirow[t]{3}{*}{ Diversos } & 107.7 & 34.90 & 1 \\
\hline & 25.47 & 8.49 & 2 \\
\hline & 6.33 & 2.11 & $3 \mathrm{at}$ \\
\hline
\end{tabular}

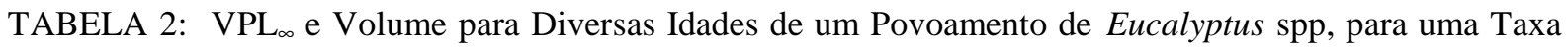
de Desconto de $8 \%$.

\begin{tabular}{c|c|c}
\hline Idade (anos) & Volume (st/ha) & VPL $_{\infty}$ (US\$/ha) \\
\hline 1 & 28,16 & $-6,219.11$ \\
2 & 44,18 & $-2,785.85$ \\
3 & 68,10 & $-1,172.02$ \\
5 & 12,11 & -72.29 \\
6 & 146,86 & 806.83 \\
7 & 198,80 & $1,470.12$ \\
8 & $\mathbf{2 4 8 , 1 9}$ & $\mathbf{1 , 8 1 8 . 9 4}$ \\
9 & 282,44 & $1,787.15$ \\
10 & 296,94 & $1,470.01$ \\
\hline
\end{tabular}

Em que: $\mathrm{VPL}_{\infty}=$ valor presente líquido considerando a replicação do projeto para um horizonte infinito. 
A época ótima de corte do povoamento foi aos 7 anos de idade, quando o VPL $L_{\infty}$ foi maior. Os dados da Tabela 2 foram considerados a base para outros cálculos. Mesmo sabendo que, do alto fuste para as talhadias, a idade de corte pode mudar, neste estudo foi considerado que ela é a mesma. A variação entre alto fuste e talhadia pode ser desprezada para fins de cálculo (Lopes, 1990). O VPL $\mathrm{V}_{\infty}$ positivo indica que o projeto é economicamente viável.

\section{Desenvolvimento teórico do modelo matemático}

Para facilitar o estudo do efeito do progresso tecnológico nos custos, será mostrado, para fins de modelagem, apenas o custo médio de implantação de 1 ha de floresta de Eucalyptus spp nas empresas que atuam na região do Cerrado do estado de Minas Gerais. Esses custos ocorrerão ao longo do tempo como no seguinte esquema:

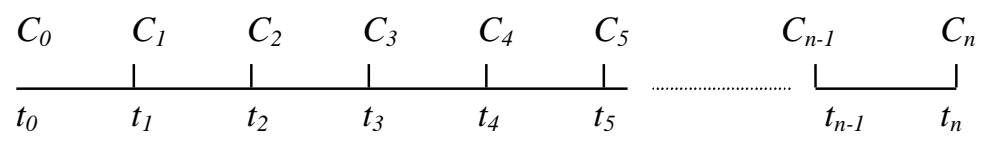

Em que: $\mathrm{C}=$ custo de implantação de 1 ha de floresta de Eucalyptus spp; $\mathrm{n}=$ número de implantações; $\mathrm{t}$ = rotação em anos.

Porém, da forma como os custos estão dispostos, eles não retratam a realidade, porque são constantes. Sabe-se que isso, na prática, não ocorreu. Beneficiados pelo progresso tecnológico, os custos sofreram redução ao longo do tempo.

Para retratar uma situação dinâmica (progresso tecnológico) que se quer, é preciso determinar a que taxa os custos vão ser reduzidos ao longo do tempo. Se eles caem, a cada implantação, não serão sempre constantes $\left(C_{0}=C_{1}=C_{2}=C_{n}\right)$ e $\operatorname{sim} C_{1}=C_{0}-\Delta C_{0}$. Este $\Delta C_{0}$ pode ser reduzido a uma taxa anual de redução no custo. Assim:

$$
C_{1}=C_{0}\left(1-h_{i}\right)^{t_{i}}
$$

Em que: $\mathrm{C}_{1}=$ custo da $1^{\underline{a}}$ implantação após o plantio original; $\mathrm{C}_{0}=$ custo de implantação original; $\mathrm{h}_{\mathrm{i}}=$ taxa anual de decréscimo de $\mathrm{C}$ no período $\mathrm{t}_{\mathrm{i}}$ (rotação);

Assim, tem-se:

$$
\begin{aligned}
& C_{1}=C_{0}\left(1-h_{1}\right)^{t 1} \\
& C_{2}=C_{0}\left(1-h_{1}\right)^{t 1}\left(1-h_{2}\right)^{t 2} \\
& C_{3}=C_{0}\left(1-h_{1}\right)^{t l}\left(1-h_{2}\right)^{t 2}\left(1-h_{3}\right)^{t 3} \\
& \mathrm{M} \\
& C_{n}=C_{0}\left(1-h_{1}\right)^{t l}\left(1-h_{2}\right)^{t 2}\left(1-h_{3}\right)^{t 3} \ldots \ldots\left(1-h_{n}\right)^{t n}
\end{aligned}
$$

Para simplificar e permitir a modelagem, assume-se que:

$$
\begin{aligned}
& h_{1}=h_{2}=h_{3}=\ldots . .=h_{n}=h \\
& t_{1}=t_{2}=t_{3}=\ldots . .=t_{n}=t .
\end{aligned}
$$

Assim:

$$
\begin{aligned}
& C_{1}=C_{0}(1-h)^{t} \\
& C_{2}=C_{0}(1-h)^{2 t} \\
& \mathrm{M} \\
& C_{n}=C_{0}(1-h)^{n t}
\end{aligned}
$$

Embora os demais custos não estejam listados, o raciocínio é idêntico. As várias situações possíveis, em que podem ocorrer as reformas, são explicitadas a seguir.

Para o caso de reforma após um corte, tem-se as seguintes expressões: 


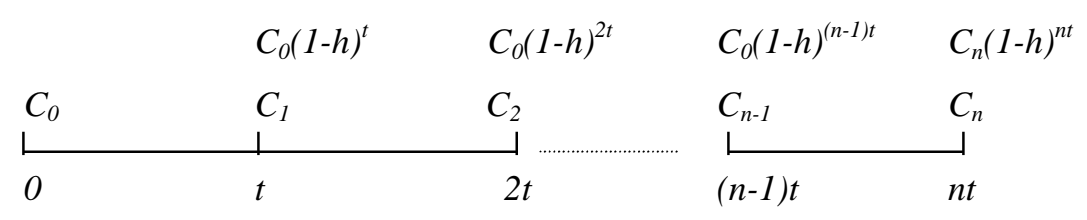

Para o caso de reforma após dois cortes, tem-se as seguintes expressões:

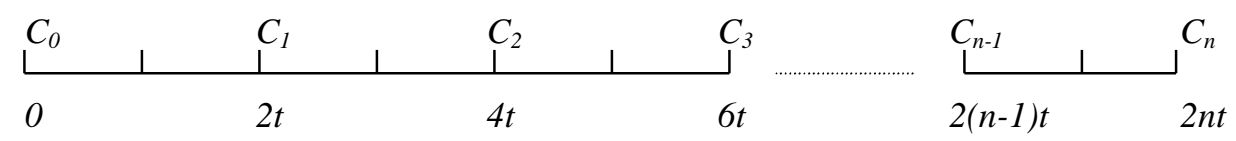

Nesta situação:

$C_{1}=C_{0}(1-h)^{2 t}$

$C_{2}=C_{0}(1-h)^{4 t}$

$C_{3}=C_{0}(1-h)^{6 t}$

$\mathrm{M}$

$C_{n-1}=C_{0}(1-h)^{2(n-1) t}$

$C_{n}=C_{0}(1-h)^{2 n t}$

Para o caso de reforma após três cortes, tem-se as seguintes expressões:

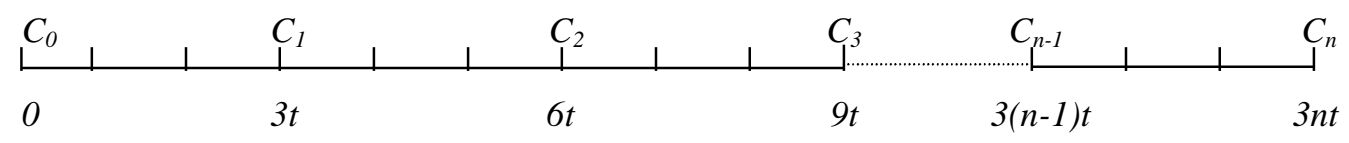

Nesta situação:

$$
\begin{aligned}
& C_{1}=C_{0}(1-h)^{3 t} \\
& C_{2}=C_{0}(1-h)^{6 t} \\
& C_{3}=C_{0}(1-h)^{9 t} \\
& \mathrm{M} \\
& C_{n-1}=C_{0}(1-h)^{3(n-1) t} \\
& C_{n}=C_{0}(1-h)^{3 n t}
\end{aligned}
$$

Para o caso de reforma após " $z$ " cortes (talhadias), tem-se as seguintes expressões:

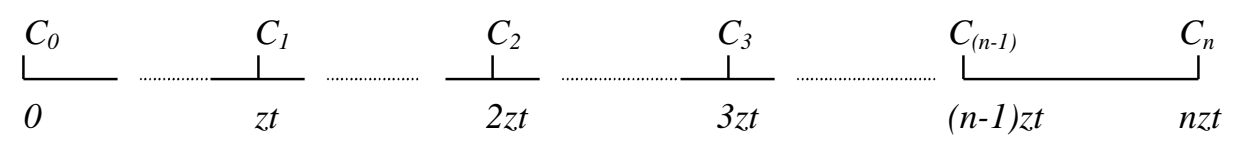

Nesta situação:

$$
\begin{aligned}
& C_{1}=C_{0}(1-h)^{z t} \\
& C_{2}=C_{0}(1-h)^{2 z t} \\
& C_{3}=C_{0}(1-h)^{3 z t} \\
& \mathrm{M} \\
& C_{n-1}=C_{0}(1-h)^{(n-1) z t} \\
& C_{n}=C_{0}(1-h)^{n z t}
\end{aligned}
$$

Da maneira como foi colocada, a equação (2) não retratará, com fidelidade, o decréscimo do custo, uma vez que a taxa "h" não apresenta o mesmo comportamento ao longo do tempo. Para resolver a situação, o uso de um moderador que, aplicado à taxa "h", permita aos custos decrescerem a uma taxa decrescente, fez-se necessário. Essa providência evita que o custo de implantação atinja valores irreais. Assim, para o caso de reforma após um corte:

$C_{0} \quad C_{1} \quad C_{2} \quad C_{3} \quad C_{n-1} \quad C_{n}$ 


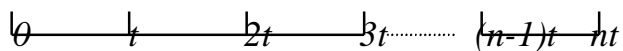

Em que:

$$
\begin{aligned}
& G_{1}=C_{0}\left[1-h(1-w)^{t}\right]^{t} \\
& C_{2}=C_{0}\left[1-h(1-w)^{2 t}\right]^{2 t} \\
& C_{3}=C_{0}\left[1-h(1-w)^{3 t}\right]^{3 t} \\
& \mathrm{M} \\
& C_{n-1}=C_{0}\left[1-h(1-w)^{(n-1) t}\right]^{(n-1) t} \\
& C_{n}=C_{0}\left[1-h(1-w)^{n t}\right]^{n t}
\end{aligned}
$$

Para o caso de reforma após dois cortes, têm-se:

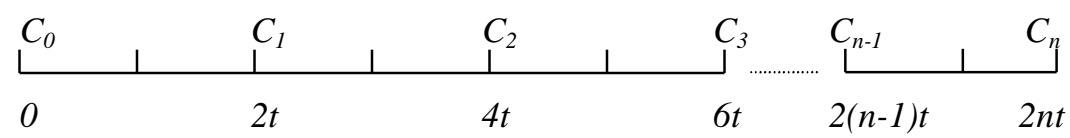

Em que:

$$
\begin{aligned}
& C_{1}=C_{0}\left[1-h(1-w)^{2 t}\right]^{2 t} \\
& C_{2}=C_{0}\left[1-h(1-w)^{4 t}\right]^{4 t} \\
& C_{3}=C_{0}\left[1-h(1-w)^{6 t}\right]^{6 t} \\
& \mathrm{M} \\
& C_{n-1}=C_{0}\left[1-h(1-w)^{2(n-1) t}\right]^{2(n-1) t} \\
& C_{n}=C_{0}\left[1-h(1-w)^{2 n t}\right]^{2 n t}
\end{aligned}
$$

Para o caso de reforma após " $z$ " cortes, têm-se:

$$
\frac{C_{0}}{0} \cdots \cdots \cdots \cdots \frac{C_{1}}{z t} \cdots \cdots \cdots \cdots \cdots \frac{C_{1}}{2 z t} \cdots \cdots \cdots \cdots
$$

Em que:

$$
\begin{aligned}
& C_{1}=C_{0}\left[1-h(1-w)^{z t}\right]^{z t} \\
& C_{2}=C_{0}\left[1-h(1-w)^{2 z t}\right]^{2 z t} \\
& C_{3}=C_{0}\left[1-h(1-w)^{3 z t}\right]^{\beta z t} \\
& \mathrm{M} \\
& C_{n-1}=C_{0}\left[1-h(1-w)^{(n-1) z t}\right]^{(n-1) z t} \\
& C_{n}=C_{0}\left[1-h(1-w)^{n z t}\right]^{h z t}
\end{aligned}
$$

Em que: "z" = número de cortes por implantação. 
A equação 3 é o modelo proposto.

Para o cálculo do valor presente dos custos, têm-se a seguinte expressão:

$$
V P C=C_{0}+C_{1}(1+r)^{-z t}+C_{2}(1+r)^{-2 z t}+C_{3}(1+r)^{-3 z t}+\ldots+C_{n}(1+r)^{-n z t}
$$

As receitas são obtidas com a venda da produção $(\mathrm{Y})$ ao preço de mercado $(\mathrm{P})$. Logo, a receita $(\mathrm{R})$ em cada corte é dada por:

$$
R=Y * P
$$

Substituindo (1) em (4) tem-se:

$$
R=K\left(1-e^{-a \cdot e^{b . m}}\right) * P
$$

Considerar a produtividade constante, é o mesmo que pressupor que as receitas serão sempre iguais. Se o preço permanecer constante, sucessivas receitas ao longo do tempo podem ser expressas, para "z" cortes e "n" implantações, como no esquema a seguir:
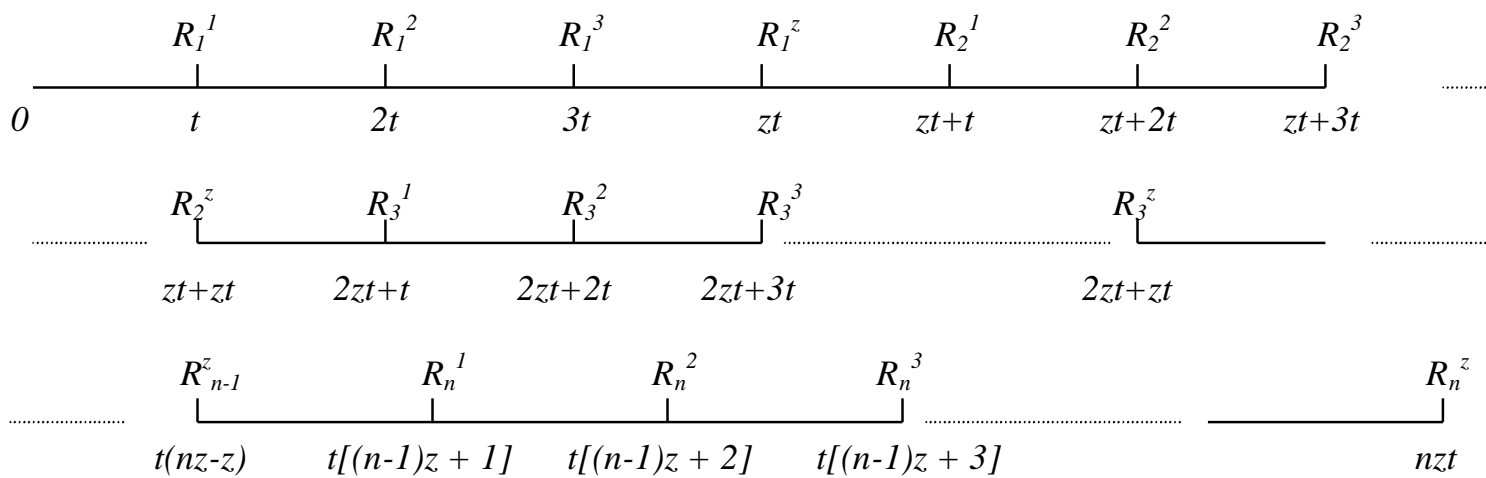

Em que:

$R_{1}{ }^{2}=\beta * R_{l}{ }^{1}$

$R_{l}^{3}=\beta^{*} R_{l}^{2}=R_{l}{ }^{l} * \beta^{2}$;

$\mathrm{E}$, assim, sucessivamente.

O fator " $\beta$ " é o fator que corrige o volume da primeira talhadia em rela ção ao volume do alto fuste e das demais talhadias em relação à talhadia anterior. O valor de " $\beta$ " foi considerado como sendo $0,90(90 \%)$ do volume anterior.

O valor atual da série representada anteriormente pode ser calculado pela seguinte express ão:

$$
\begin{aligned}
& V P(R B)=\left\{R_{1}^{1}(1+r)^{-t}+R_{1}^{2}(1+r)^{-2 t}+\ldots .+R_{1}^{z}(1+r)^{-z t}+R_{2}^{1}(1+r)^{-t(z+1)}+\right. \\
& +R_{2}^{2}(1+r)^{-t(z+2)}+\ldots .+R_{2}^{z}(1+r)^{-2 z t}+\ldots .+R_{n-1}^{z}(1+r)^{-t(n z-z)}+R_{n}^{2}(1+r)^{-t[(n-1) z+1]}+\ldots \\
& +R_{n}^{2}(1+r)^{\left.-t[(n-1) z+1]_{+}+\ldots+R_{n}^{z}(1+r)^{-z n t}\right\}}
\end{aligned}
$$

Em que:

$\mathrm{R}_{1}{ }^{1}$ é a receita bruta do $1^{\mathrm{o}}$ corte da $1^{\mathrm{a}}$ implantação.

$\mathrm{R}_{1}^{2}$ é a receita bruta do $2^{\mathrm{o}}$ corte da $1^{\mathrm{a}}$ implantação.

$M$

$\mathrm{R}_{\mathrm{n}}{ }^{\mathrm{z}}$ é a receita bruta do z-ézimo corte da n-ézima implantação.

\section{Aplicação prática do modelo matemático}

Para testar a validade do modelo, foram calculados, como exemplo, os valores dos custos de 
implantação em 1 ha de florestas de Eucalyptus spp na região do cerrado de Minas Gerais, ao longo das décadas de 60 a 90 e, posteriormente, projetados para o período entre 2000 e 2040. Como ponto de partida foi considerado o custo de implantação adotado pelo IBDF (1974) à época dos incentivos fiscais que era de US $\$ 1,800.00 /$ ha. De posse dessa informação e com os valores atuais, foi calculada a taxa média de decréscimo " $h$ " dos custos ao longo do período considerado. Porém, como já foi comentado, a taxa "h" não diminuirá indefinidamente e de modo constante ao longo do tempo. A queda será cada vez menor, havendo uma estabilização no futuro. Assim, será introduzida a taxa "w" que suavizará a queda de "h" no tempo. Para o cálculo dos demais custos o raciocínio é idêntico.

Com base no modelo proposto, foram calculados os custos de implantação representados na Tabela 3 , tendo sido observado que os custos tiveram quedas mais bruscas na primeira parte do estudo, ou seja, entre 1960 e 2000.

TABELA 3: Redução do Custo de Implantação do hectare (ha) de Florestas de Eucalyptus spp na Região do Cerrado do estado de Minas Gerais.

\begin{tabular}{c|c|c|c}
\hline \multicolumn{2}{c|}{$1^{-}$Parte do estudo } & \multicolumn{2}{c}{$2^{2}$ Parte do estudo } \\
\hline Década & Custo (US\$/ha) & Década & Custo (US\$/ha) \\
\hline 1960 & $1,800.00$ & 2000 & 600.00 \\
1970 & $1,337.75$ & 2010 & 542.89 \\
1980 & $1,009.42$ & 2020 & 491.67 \\
1990 & 772.86 & 2030 & 445.71 \\
2000 & 600.00 & 2040 & 404.42 \\
\hline
\end{tabular}

A própria trajetória do progresso tecnológico explica as diferenças maiores nos primeiros 40 anos estudados. As taxas de progresso tecnológico vão sendo cada vez menores ao longo do tempo. Quando se conseguem grandes reduções como no início do período considerado, pode-se dizer que a tecnologia anterior era ainda incipiente.

Observa-se que a queda nos custos foi estudada dentro de um horizonte de 40 anos. Tal fato se deve às próprias limitações apresentadas pelo modelo. A parte do modelo $\left[1-h(1-w)^{z t}\right]^{z t}$ cresce até um certo ponto com "zt", posteriormente, ela começa a cair, provocando aumentos nos custos. Essas restrições do modelo são mais pronunciadas quanto maiores forem os valores de "h" e "w". Tais valores foram $\mathrm{h}=3 \%$ e $1 \%$ e $\mathrm{w}=0,26 \%$ e $0,05 \%$ para o período de 1960/2000 e 2000/2040 respectivamente.

A Figura 2 mostra a tendência do comportamento dos custos nos dois períodos estudados.

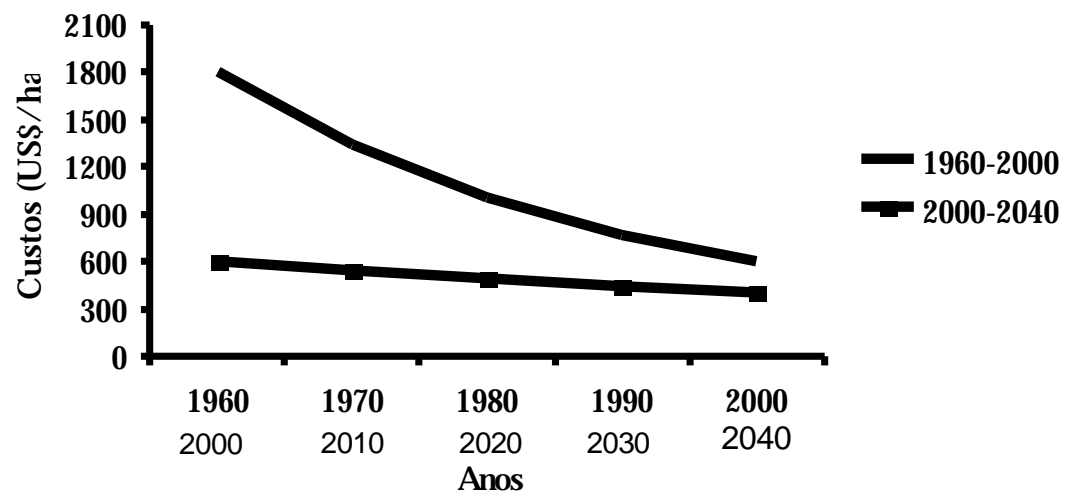

FIGURA 2: Decréscimo dos Custos de Implantação do ha de Florestas de Eucalyptus spp no Cerrado do estado de Minas Gerais.

Embora os valores retratem a situação do Cerrado do estado de Minas Gerais, não fogem muito da média de custos considerando o Brasil como um todo. 


\section{Cadeia de Substituição}

O estudo da cadeia de substituição foi realizado em etapas. Na primeira, foi estudado o efeito do progresso tecnológico na época ótima de substituição considerando os custos decrescentes e a receita constante desde a década de 60 . Na segunda etapa foram projetadas as épocas ótimas de substituição para 40 anos, dada uma taxa de progresso tecnológico sobre os custos e as receitas mantidas constantes em valores atuais (1999).

\section{Período de 1960-2000}

Os dados de custos para a década de 60 foram considerados como três vezes maiores que os atuais, exceção feita ao valor da terra que foi de US\$200.00/ha (Souza,1999), considerado como o custo de oportunidade da terra juros à taxa de $8 \%$ a.a., sendo, portanto, um custo anual. A Tabela 4 retrata como seria a cadeia de substituição se as receitas permanecessem as mesmas desde o in ício do período considerado, sendo apenas os custos beneficiados pelo progresso tecnológico.

TABELA 4: Número de cortes que antecederiam às substituições de povoamentos de Eucalyptus spp se as receitas fossem as mesmas desde a década de 60 .

\begin{tabular}{c|c|c}
\hline Década & VPL $_{\infty}($ US $\$ /$ ha $)$ & N. de Cortes \\
\hline $1960-1970$ & $-2,072.63$ & 17 \\
$1970-1980$ & $-1,369.49$ & 11 \\
$1980-1990$ & -864.33 & 8 \\
$1990-2000$ & -496.69 & 6 \\
\hline
\end{tabular}

Em que: $\mathrm{VPL}_{\infty}=$ valor presente líquido considerando a replicação do projeto para um horizonte infinito.

Os valores mostram que, se de fato somente os custos tivessem sido beneficiados pela tecnologia, até hoje, as empresas trabalhariam com prejuízos, caminhando para uma condição em que se esperassem lucros na atividade. Na realidade, houve "progresso tecnológico" tanto nos custos (redução) quanto na produtividade (aumento). Observou-se que se o custo diminui, o número de cortes para o caso onde o VPL é negativo também diminui e quanto mais rápido o VPL se aproximar de valores positivos, mais rapidamente cairá o número de cortes.

Em trabalho semelhante, Rezende et al. (2001) estudaram a reforma para o caso do aumento das receitas beneficiadas pelo avanço tecnológico na produtividade. Os autores concluíram que houve queda no número de cortes entre implantações em conseqüência do progresso tecnológico, e que, para o caso estudado, o número de cortes que maximiza o lucro atualmente é 2, podendo passar para 3 cortes se houver aumento na produtividade das talhadias.

\section{Período de 2000-2040}

Partindo do pressuposto de que as receitas estariam chegando a um ponto no tempo em que não mais aumentariam, e somente os custos seriam reduzidos pelo progresso tecnológico, a segunda parte do estudo procurou mostrar como seria a cadeia de substituição para os próximos 40 anos. A Tabela 5 apresenta os valores encontrados para esse período.

TABELA 5: Número de cortes que antecederão à substituição de povoamentos de Eucalyptus spp no estado de Minas Gerais.

\begin{tabular}{c|c|c}
\hline Década & VPL $_{\infty}($ US\$ $/$ ha $)$ & N. de Cortes \\
\hline $2000-2010$ & $2,995.53$ & 2 \\
$2010-2020$ & $3,285.69$ & 2 \\
$2020-2030$ & $3,545.78$ & 2 \\
$2030-2040$ & $3,835.18$ & 2 \\
\hline
\end{tabular}

Em que: $\mathrm{VPL}_{\infty}=$ valor presente líquido considerando a replicação do projeto para um horizonte infinito.

Observou-se que a substituição será após dois cortes nos próximos 40 anos. É importante ressaltar que os resultados se aplicam a uma situação de empresas que atuam em áreas de Cerrado do estado de Minas Gerais. 
Aumentos em variáveis como taxa de desconto, produtividade das talhadias $(\beta)$, preço da madeira e custo de exploração tendem a elevar a idade de substituição (o número de cortes), e aumentos na produtividade tendem a reduzi-la.

No caso estudado, havendo progresso tecnológico em $\beta$ que permita elevar sua produtividade para 93\% em relação ao alto fuste, o número de cortes passará para três e quando o valor de $\beta$ tender a 1 , o número de cortes tenderá ao infinito.

\section{CONCLUSÕES}

Os resultados alcançados e as análises realizadas permitiram as seguintes conclusões:

a) A rotação florestal com valores atuais (1999) encontra-se aos 7 anos de idade.

b) Mantidas as receitas da década de 60, a idade ótima de substituição estaria após seis cortes.

c) O modelo matemático proposto mostrou-se eficiente para estimativas e previsões em horizontes de até 40 anos.

d) As épocas ótimas de substituição caíram ao longo dos anos, passando dos seis cortes na década de 60 para três cortes na década de 80 , chegando atualmente à substituição após dois cortes.

e) Para os futuros povoamentos, verificou-se uma tendência de permanecer nos dois cortes antes da substituição, podendo passar a três cortes se a tecnologia conseguir um pequeno aumento na produtividade das talhadias.

\section{AGRADECIMENTO}

Os autores agradecem à Fundação de Amparo à Pesquisa do Estado de Minas Gerais (FAPEMIG) pelo apoio financeiro.

\section{REFERÊNCIAS BILBIOGRÁFICAS}

IBDF. Zoneamento econômico florestal do Estado de Minas Gerais. Belo Horizonte-MG, 1974. 182p.

LOPES, H.V.S. Análise econômica dos fatores que afetam a rotação de povoamentos de eucaliptos. 1990. 188p. Dissertação (Mestrado) - Universidade Federal de Viçosa, Viçosa.

MASSÉ, P. Optimal investiment decisions rules for action and criteria choice. Prentice. Hall, 1962. 500 p.

REZENDE, J.L.P.; OLIVEIRA, A. D. Análise econômica e social de projetos florestais. Viçosa: Universidade Federal de Viçosa, 2001. 389p.

REZENDE, J.L.P.; PAULA Jr., G.G.; RIBEIRO, G.A., Técnicas de análise econômicas usadas na tomada de decisão referentes à reforma de eucaliptais. In: SEMINÁRIO SOBRE ASPECTOS TÉCNICOS E ECONÔMICOS DA REFORMA DE EUCALIPTAIS, 1987, Belo Horizonte. Anais ... Belo Horizonte: UFV/SIF, 1987.

REZENDE, J.L.P.; SOUZA, A.N.; OLIVEIRA, A.D. Estudo do momento ótimo de substituição de povoamentos de Eucalyptus spp: o caso do aumento das receitas. Rev. Scientia Forestalis, Piracicaba, n. 59, p.27-39, jun. 2001.

SILVA, A.A.L., Análise econômica da substituição de povoamentos de Eucalyptus spp. 1990. 109p. Dissertação (Mestrado) - Universidade Federal de Viçosa, Viçosa.

SOUZA, A.N. Estudo econômico da reforma de povoamentos de Eucalyptus spp. - O caso do progresso tecnológico. 1999. 140p. Dissertação (Mestrado) - Universidade Federal de Lavras, Lavras.

STEINDL, J. Progresso técnico, distribuição e crescimento: progresso técnico e teoria econômica. São Paulo: Hucitec, 1980. 183p. (Economia e Planejamento, Série Técnica Contemporânea). 
